\section{Dynamics of a Radially Rotating Beam With Impact, Part 2: Experimental and Simulation Results}

A theoretical and computational model to study the dynamics of a radially rotating beam with impact were presented in Part 1. In this paper the experimental results are presented and they are compared with simulations using the momentum balance model described in Part 1. Excellent agreement was found between the experiments and simulation. Sensitivity studies were employed to show that the model is applicable for a fairly wide range of parameter values. Thus, the momentum balance method has been demonstrated to be capable of accurately predicting the dynamics of systems which consist of rigid and elastic links undergoing impact.
A. S. Yigit

Lecturer.

A. G. Ulsoy

Associate Professor.

R. A. Scott

Professor.

Department of Mechanical Engineering and Applied Mechanics, The University of Michigan, Ann Arbor, Michigan 48109

\section{Introduction}

A theoretical and computational model using the momentum balance (coefficient of restitution) method was presented in Part 1 (Yigit et al., 1988a). In this paper experimental and numerical studies on this subject are presented. Experimental results are compared with simulation results in order to assess the validity of the model.

The objective of the experimental work is to investigate the validity of the analytical impact model presented in Part 1, and to determine its applicability for flexible systems. This task is undertaken by considering a radially rotating flexible beam, with a rigid stem, impacting a small semi-cylindrical hard surface. Three types of measurements are made: torque input, rigid body motion, and elastic motion of the flexible beam. The torque input given to the shaft by a dc motor was measured through a current measurement. The angular velocity of the rigid stem was measured by using an optical incremental encoder. Electrical resistance strain gages at four different locations along the beam were used to obtain the elastic motion. The measured angular velocity of the rigid shaft and the strains at four different locations were compared with simulations using a torque input approximating the measured torque. Also a set of experiments were performed with no torque input, and the natural frequencies of the nonrotating and rotating beam were obtained. High speed video recordings were employed to show the existence of multiple impacts.

Experimental results are compared with simulation results in order to assess the validity of the model. Sensitivity studies are given to determine the applicability of the model for a wider range of system parameters than covered by the experiments.

In Section 2 the experimental design used is presented. The calibration procedure for the instrumentation is explained in
Section 3. Experimental and simulation results are given and compared in Section 4. Section 5 is devoted to sensitivity studies. Finally some concluding remarks are given in Section 6.

\section{Experimental Design}

The schematic diagram of the experimental setup is shown in Fig. 1. The individual components in the setup are described below.

- Flexible Beam: A slender aluminum bar with the following dimensions and properties was selected: length $=.530 \mathrm{~m}$, diameter $=.0063 \mathrm{~m}$, mass density $=2700 \mathrm{~kg} / \mathrm{m}^{3}$, Young's modulus, $\mathrm{E}=6.89510^{10} \mathrm{~N} / \mathrm{m}^{2}$. The beam is cantilevered on a rigid shaft, and was designed so that the natural frequencies are small and at the same time the deflections under the impact are within the elastic limit. Capture of the third mode contribution was to be within the range of the measurements. The specifications given above satisfy these requirements.

- DC Motor: The dc motor used for driving the system was an Electro-Craft-Model $M-1030$ which is an intermediate performance dc moving coil servomotor. The damping factor is $410^{-4} \mathrm{Nms}$ and the minimum MotorTach resonant frequency is $4 \mathrm{kHz}$. The magnitude of the torque which is delivered by the dc motor was controlled using an adjustable voltage and current power supply.

- Encoder: In order to measure the rigid shaft angular velocity an optical incremental encoder was used. The unit was Model 25G Instrument Grade by Sequential Information Systems, Inc. Since the shaft turns only about 180 deg. during the experiments, a high resolution encoder was necessary to get high bandwidth velocity measurements. This encoder has a disk resolution of 16,384 pulses/revolution. Its starting torque is $7.0610^{-3} \mathrm{Nm}$, its slew speed is $500 \mathrm{rad} / \mathrm{s}$ max., its maximum angular acceleration is $100,000 \mathrm{rad} / \mathrm{sec}^{2}$, and its frequency response 


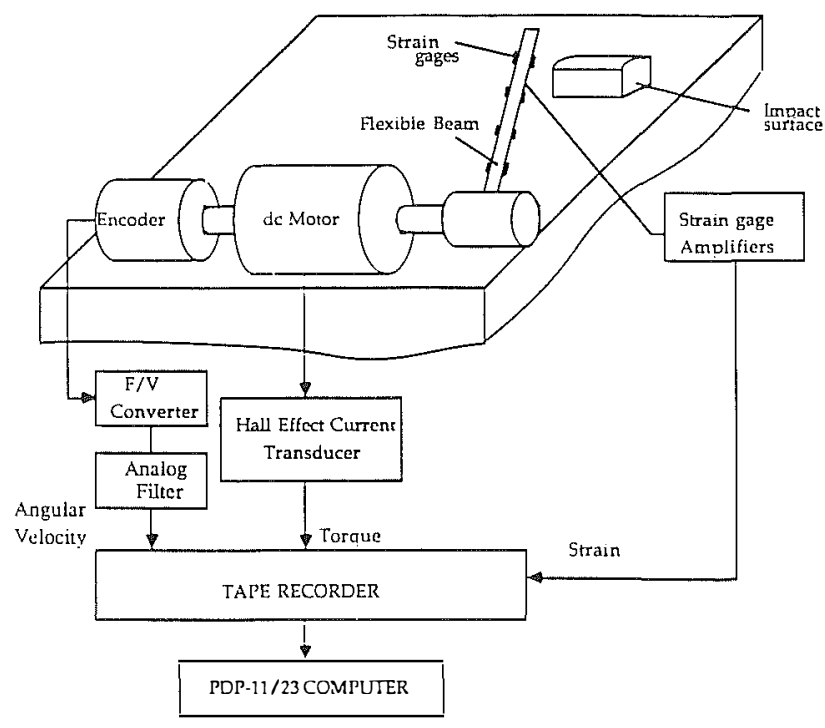

Fig. 1 The sketch of the experimental setup

is $50 \mathrm{kHz}$. Accuracy (pulse to any other pulse) is \pm 1.0 minutes of arc. The encoder produces pulses from two different channels for clockwise and counterclockwise rotations.

- Frequency-to-Voltage Converter: An Analog Devices Model AD650 Frequency-to-Voltage Converter is used to convert the encoder pulse frequency into an analog signal. The unit is capable of doing frequency-to-voltage and voltage-to-frequency conversions for frequencies up to $1 \mathrm{MHz}$. The typical nonlinearity at $100 \mathrm{kHz}$ is 0.005 percent, and it can accept input pulses of duration greater than 100ns. Two separate converters had to be used for the two different channels from the encoder because the frequency-to-voltage converter output is always positive. Then these two outputs were summed digitally in the computer.

- Current Transducer: The torque delivered to the rigid shaft by the dc motor was measured using a Model CT100LTT current transducer by Ohio Semitronics which measures the current using the Hall effect (Walden). This transducer provides more accurate and safer measurements than conventional shunt measurements. The bandwidth is 0 to $5 \mathrm{kHz}$., linearity is 0.5 percent full scale, and its response time is less than $50 \mu \mathrm{sec}$. The dc signal conditioner used with the unit was Model CTA101 Ohio Semitronics. Its frequency range is also 0 to $5 \mathrm{kHz}$., and 40 $\mu \mathrm{sec}$ is required to reach 90 percent of the steady state value.

- Straingages and Straingage Amplifier: Strains at four locations along the beam are measured using MicroMeasurements EA 13-120LZ-120 straingages. The gage factor is $2.095 \pm 0.5$ percent. These straingages have an excellent frequency response up to $50 \mathrm{kHz}$, and they have been tested for dynamic measurements (Hetényi, 1950). A half bridge circuit arrangement was used employing two straingages at a given location on the upper and lower surfaces of the beam. Self-temperature compensation is provided. A Model 1020 Micro-Measurements Straingage Amplifier is used for the straingage instrumentation. Straingages were preferred to accelerometers because of the presence of the impact. An accelerometer which can withstand the impact is not generally capable of measureing the smaller magnitudes of the vibration before and after the impact.

- Analog Filter:The output signals from the frequency-tovoltage converters contained high frequency noise in-
Table 1 Dimensions and structural properties in the experimental setup

\begin{tabular}{|l|l|}
\hline Mass per unit length, $\rho$ & $0.0855 \mathrm{~kg} / \mathrm{m}$ \\
Flexural rigidity, $E I$ & $5.50 \mathrm{Nm}^{2}$ \\
Length of the flexible beam, $L$ & $0.530 \mathrm{~m}$ \\
Length of the root, $a$ & $0.012 \mathrm{~m}$ \\
Location of the impact, point, $x_{p}$ & $0.515 \mathrm{~m}$ \\
Inertia of the flexible beam, $I_{B}$ & $0.0042 \mathrm{kgm}^{2}$ \\
Total Inertia of the rigid shaft & \\
(including motor and encoder shafts), $I_{R}$ & $8.610^{-5} \mathrm{kgm}^{2}$ \\
Inertia ratio, $\beta=I_{R} / I_{B}$ & 0.002 \\
Modal damping for the flexible beam & 0.0 \\
Joint viscous damping coefficent, $c$ & $0.00537 \mathrm{kgm}^{2} / \mathrm{s}$ \\
\hline
\end{tabular}

troduced by the converter itself. In order to eliminate this noise, an analog filter was used with a cutoff frequency of $3 \mathrm{kHz}$. The maximum frequency in the velocity measurement corresponding to the third mode was about $200 \mathrm{~Hz}$, so this filtering has no significant effect on the measurement itself. The analog filter was a Khron-Hite Digitally Tuned 3320 Series Low Pass Filter which had an adjustable cut off frequency limits from $0.001 \mathrm{~Hz}$ to 99.9 $\mathrm{kHz}$.

- Instrumentation Tape Recorder: Data was first recorded on an analog tape recorder to avoid the sampling time restrictions of digitizing. The instrumentation tape recorder was a Model Store 7DS Racal Tape Recorder. This tape recorder has 7 data channels which have the capability of recording signals of up to $40 \mathrm{kHz}$ in frequency, depending on the recording speed and mode. In our case, FM recording was used and the speed at which the signals were recorded was $60.0 \mathrm{in} / \mathrm{sec}$, thus achieving a bandwidth of 0 to $20 \mathrm{kHz}$. Overall system linearity is 0.3 percent, signal to noise ratio is $52 \mathrm{db}$ and the harmonic distortion is less than 1.2 percent at the maximum modulation level.

- Computer: The collected data were digitized on a DEC PDP-11/23 computer by playing the tape recorder back at a slower speed, thus allowing a much longer actual sampling rate. The effective sampling rate was $1 \mathrm{kHz}$, corresponding to an actual sampling rate of $62 \mathrm{~Hz}$.

Dimensions and structural properties are presented in Table 1. The damping in the rigid shaft joint was calculated as follows: The rigid shaft (without the flexible beam mounted) was rotated with a constant angular velocity, then the motor stopped. The decay of the angular velocity was recorded on a digital storage oscilloscope. The time constant of the system was obtained neglecting the compliance in the motor shaft. The angular velocity of the shaft can be written as:

$$
\dot{\theta}=\dot{\theta}_{0} e^{-(t / \tau)}
$$

where the time constant $\tau$ can be expressed as:

$$
\tau=I / c
$$

where $I$ is the inertia of the shaft and $c$ is the viscous damping coefficent. Then knowing the time $\tau$ for the angular velocity to drop to a value which is 33 percent of the initial value, the 
viscous damping coefficient can be obtained from equation (2).

\section{Calibration}

Straingage Calibration: The strain measurement was calibrated statically. The beam was mounted as a cantilever beam and loaded statically by a series of known weights. Measured strains were compared with the calculated strains.

Torque Measurement Calibration: A balance scale was used to calibrate the system. The dc motor was turned on with the tip of the beam touching the pan of the scale. Then measuring the force, the applied torque was calculated and compared with the measurements of the current transducer. Very short response time and very low inertia of the components in the system justify the static calibration procedure.

Velocity Measurement Calibration: The optical encoder produces 16,384 pulses per revolution, which means that a frequency of $16,384 \mathrm{~Hz}$ would correspond to an angular velocity of $2 \pi[\mathrm{rad} / \mathrm{s}]$. Thus the only calibration necessary was that associated with the frequency-to-voltage converters. This was done using a function generator. The calibration curves for all measurements, and the results of linear regression analyses for these curves are given in Yigit (1988).

\section{Experimental Results and Comparison}

A set of experiments were performed and recorded using the KODAK Ekta-Pro high speed video recording system. Two separate cameras were used to focus on both the entire beam and on the impact region. The camera focused on the entire beam was intended to capture the mode shapes of the beam during its vibration, and the impact point was studied to record multiple impacts clearly. In the first set of recordings only the impact region was studied. As a second trial the screen was divided into two to show both the entire beam and the impact region. In these experiments the recording speed was 1000 frames/second. The mode shapes of the beam can be seen by playing the recordings back at a slower speed (30 frames/second). In order to see more clearly the multiple impacts the screen was divided into three and the images from the same camera were recorded on one frame thus achieving a recording speed of 3000 frames/second. Multiple impacts were clearly observed in these recordings (Yigit, 1988).

4.1 Results Without Torque Input. Some preliminary simulations were run to test accuracy. For the experiment the rigid shaft is held fixed so that a cantilever configuration is obtained. The tip of the cantilever is given a vertical impulse and the strains at four different locations along the beam are measured. The structural damping present in the flexible beam is seen to be negligible, and it is assumed to be zero for the simulations later. In order to determine the natural frequencies a discrete Fourier transform (DFT) is applied to the time response, and the first natural frequency was obtained to be 17 $\mathrm{Hz}$. Almost identical results were obtained from the other three locations. Fig. 2(a) shows the free response of the cantilever beam at location 3 . This result was obtained using seven modes in the simulation. The fundamental frequency is $18 \mathrm{~Hz}$, and this agrees well with the exact value. Experiments give a fundamental frequency of $17 \mathrm{~Hz}$ [Fig. 2(b)]. The small difference is probably due to the fact that the ideal cantilever boundary conditions were not achieved in the experimental setup.

Secondly, the beam was considered to fall freely from an initial angle $\theta_{0}$, and impact on an aluminum surface. In the simulation, the coefficient of restitution is specified as a function of impact velocity. For aluminum, for impact velocities up to $0.2[\mathrm{~m} / \mathrm{s}]$ experimental data is available (Goldsmith, 1960). For impact velocities higher than $0.2[\mathrm{~m} / \mathrm{s}]$ the value of
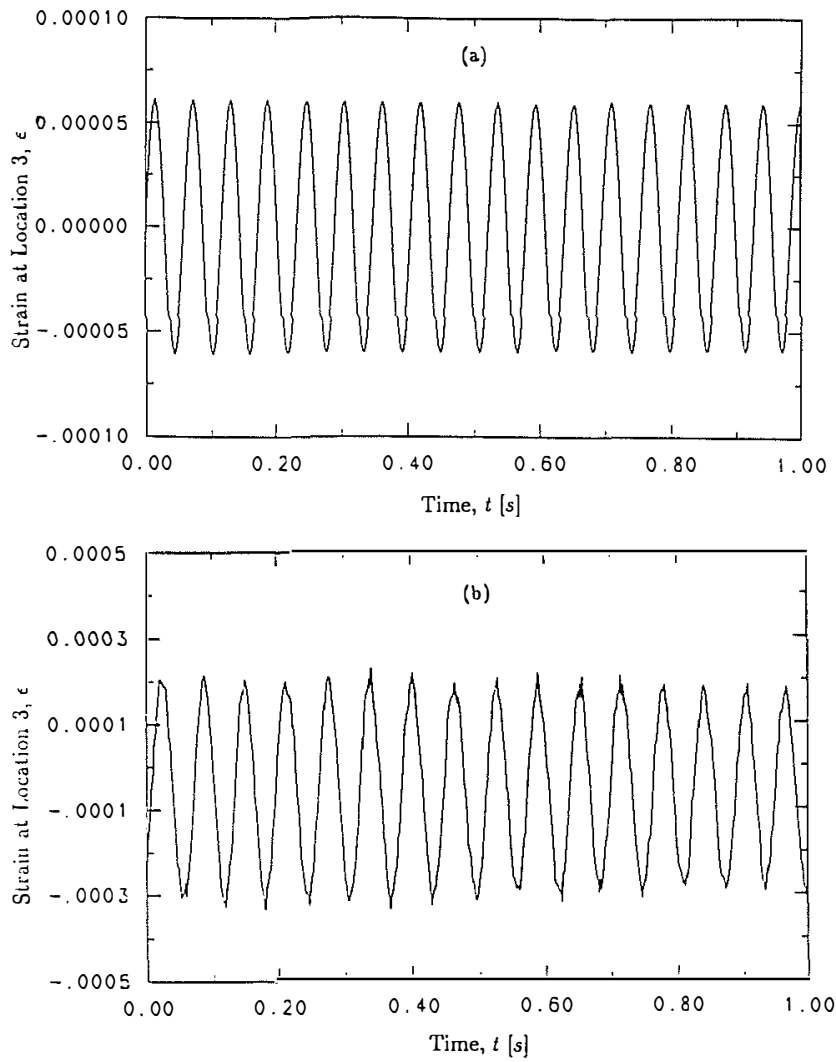

Fig. 2 Free response of the cantilever beam, (a) simulation, (b) experiment

$e$ was determined from the following analytical result (Johnson, 1985):

$$
e=0.501 v^{-(1 / 4)}
$$

Simulation and experimental results on angular velocities and strain histories are shown in Figs. 3 and 4. Peak strains agree well as can be seen by comparing Figs. $4(a)$ and $4(b)$. Due to the Coulomb friction at the joint (not modeled in the simulation) there is a time delay in the experimental results. This effect can be observed by comparing Figs. $3(a)$ and $3(b)$. The effect of Coulomb friction is seen only at the beginning of the motion where the angular velocity obtained from the simulation shows a much higher negative peak at the time of impact than the one observed in the experiment. This difference is probably due to the fact that the angular acceleration for this region is beyond the limit of the incremental optical encoder which was used to measure the angular velocity. The average acceleration at the time of impact as calculated from the simulation is $110,000\left[\mathrm{rad} / \mathrm{s}^{2}\right]$, whereas the maximum acceleration for the encoder is $100,000\left[\mathrm{rad} / \mathrm{s}^{2}\right]$. The negative peaks for the subsequent impacts are captured accurately since they fall within the acceleration limit of the encoder.

Discrete Fourier transforms show that the dominant frequency predicted by the simulation is about $67 \mathrm{~Hz}$, compared to $66 \mathrm{~Hz}$ in the experiment [note that the frequency of the rotating beam is higher than the cantilever frequency of $17 \mathrm{~Hz}$, due to the coupling between the elastic and the rigid body motions, as discussed in Yigit et al. (1988b)].

It is interesting to note that both the strain and angular velocity results show the existence of multiple impacts. This is best seen in Fig. 5, which is a magnification of the first contact/rebound phase. This phenomenon of multiple impacts has also been reported by other researchers (e.g., Goldsmith, 

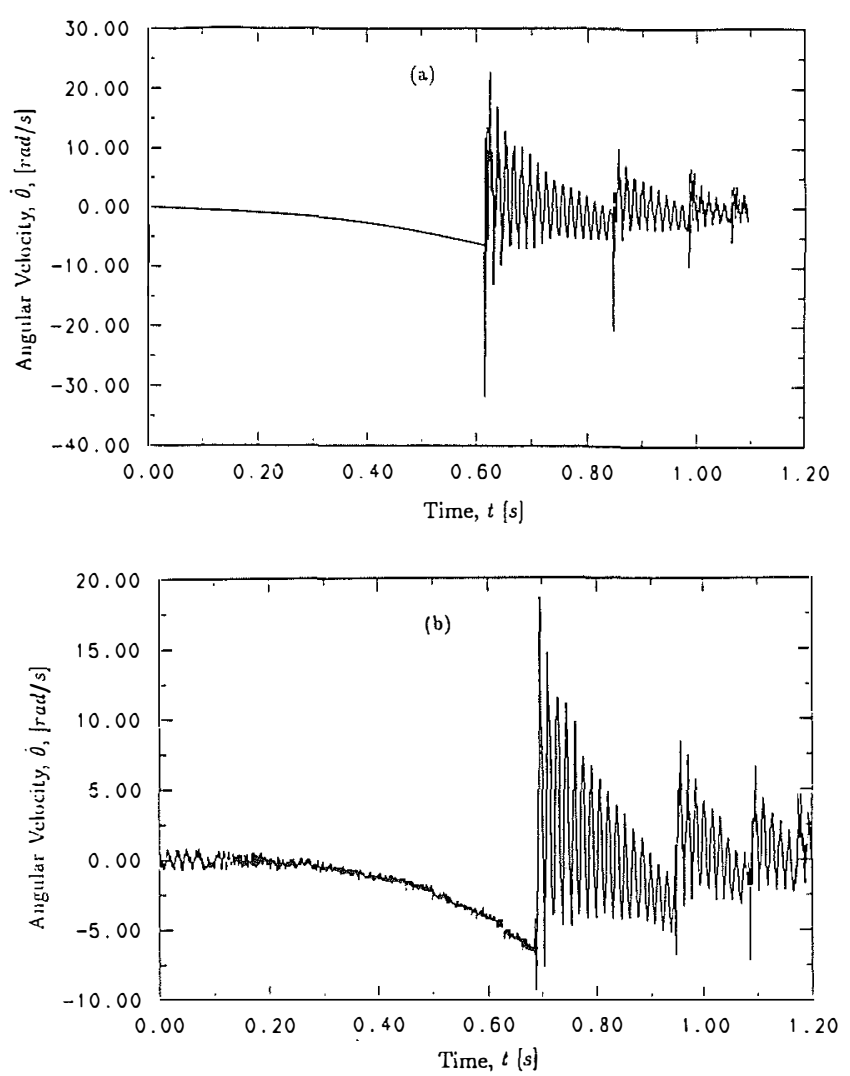

Fig. 3 Angular velocity of the rigid shaft, free fall (a) simulation, (b) experiment
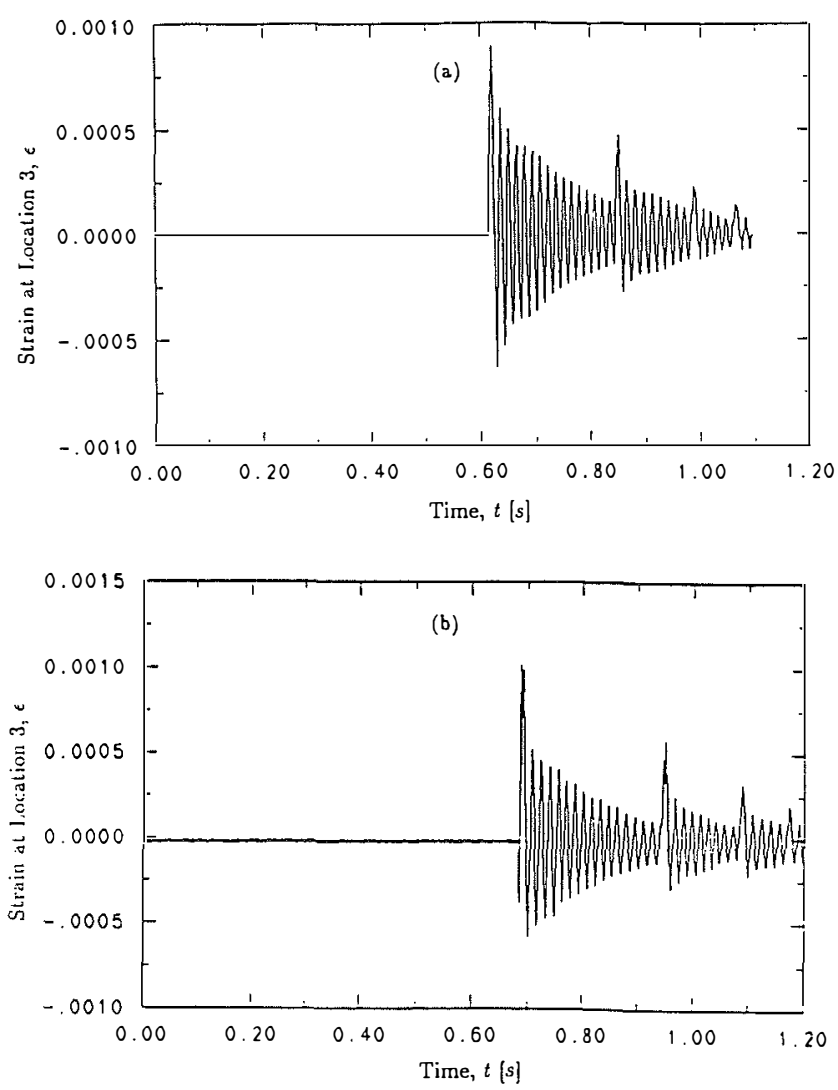

Fig. 4 Strain history at location 3 , free fall, (a) simulation, (b) experiment

1960) and are a peculiar characteristic of impacts involving flexible systems. The existence of these multiple impacts was

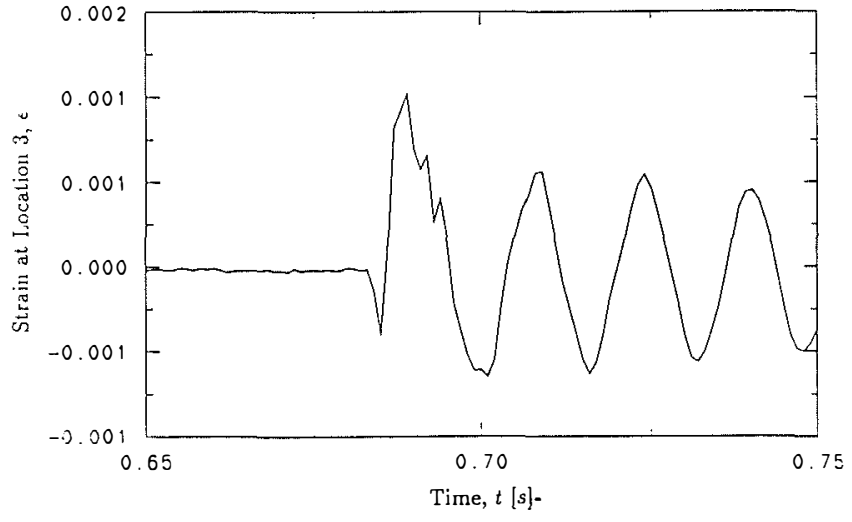

Fig. 5 Strain history, impact on aluminum surface, magnified for the impact region

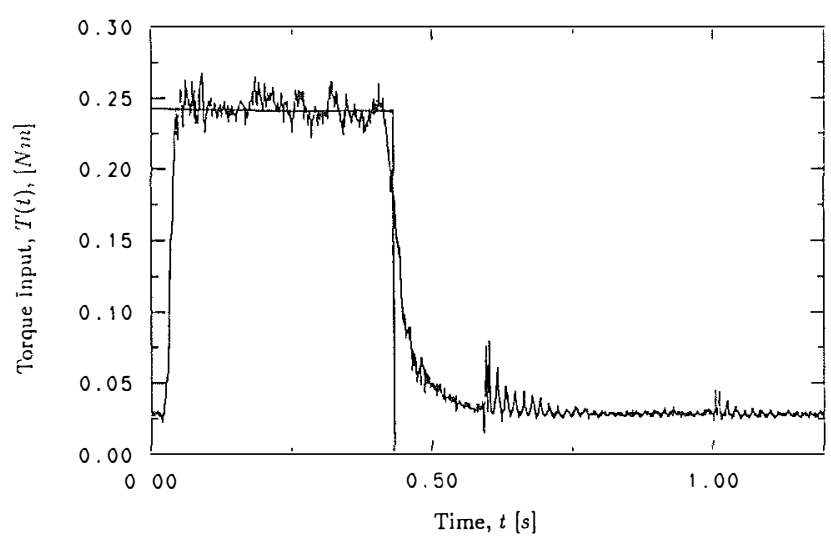

Fig. 6 Measured torque input, and approximated torque pulse for simulation

confirmed by high speed video recordings, as discussed in the previous section.

4.2 Results with Torque Input. The more challenging case of a torque is now examined. The beam impacts an aluminum surface, and the rigid shaft is given a rectangular torque pulse input which approximates the torque input in the corresponding experiments (see Fig. 6). Simulation and experimental results on angular velocities and strain histories are shown in Figs. 7 and 8. Some differences between the experiment and simulation are seen in the angular velocity results [(Figs. 7(a) and 7(b)]. The time delay in the experimental results is due to the coulomb friction which is effective during the initial period of the motion when the angular velocity is low. The negative peaks is the simulation differ with those in the experiment because of the limitation on the maximum angular acceleration of the encoder. Some other possible sources for the differences include: ( 1 ) using a rectangular profile for the torque input. As can be seen from Fig. 6 the actual torque input does not drop to zero instantaneously. This contributes to the difference in the slopes of the angular velocity just before the impact, (2) neglecting the coulomb friction in the joint: the coulomb friction becomes effective at low angular velocities. It tends to delay the time of the first impact, and it causes the second impact after bouncing back to occur earlier than predicted by the simulation. Excellent agreement is found for the peak strains [compare Figs. 8(a) and $8(b)$ ]. Strains at the other three locations exhibit a very similar behavior. The momentum balance method appears to give very good results in terms of the elastic motion.

Another simulation was performed in which the value of the coefficient of restitution was determined based on the velocity of the first impact and held constant throughout the simulation. Results are presented in Figs. 9 and 10 for the angular 

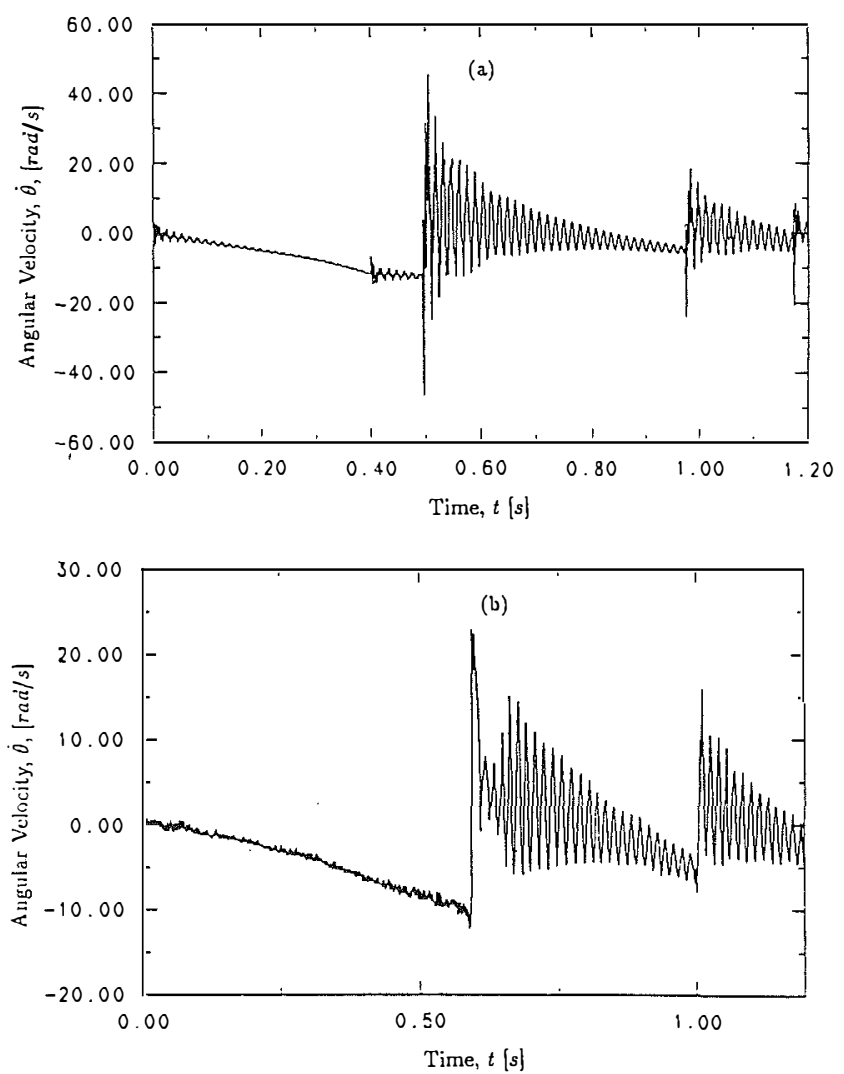

Fig. 7 Angular velocity of the rigid shaft, (a) simulation, (b) experiment
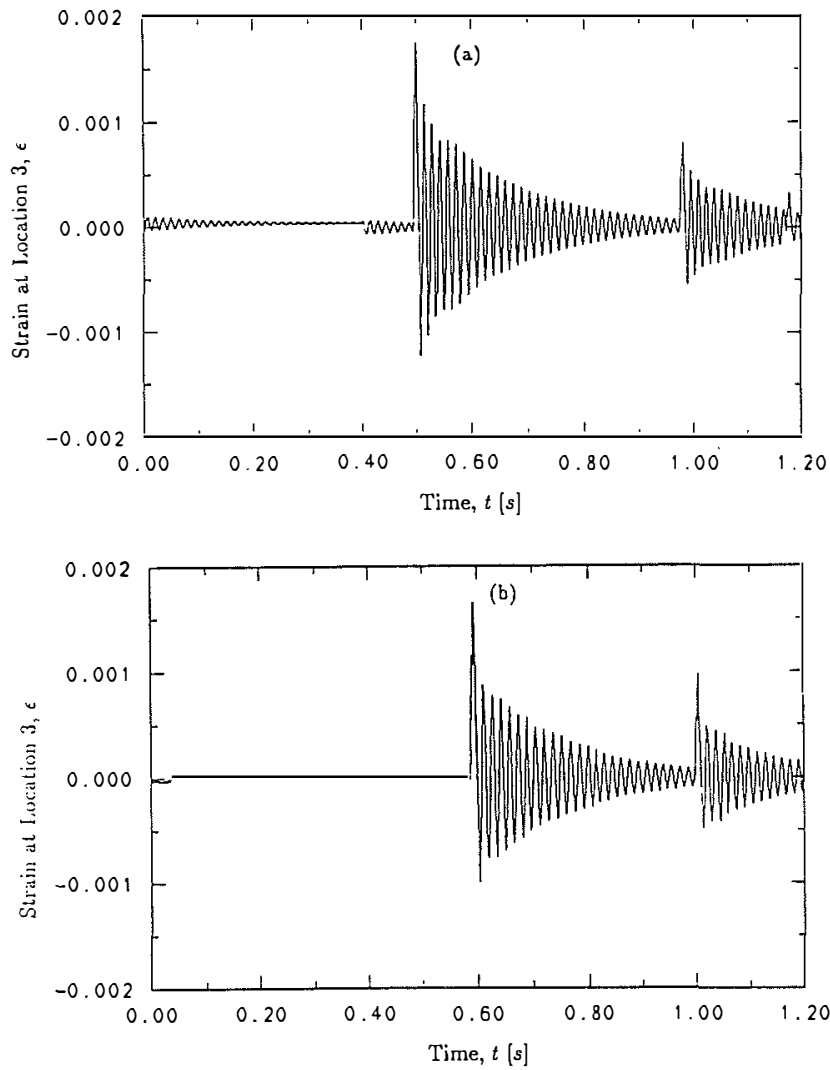

Fig. 8 Strain history at location 3, (a) simulation, (b) experiment

velocity of the rigid shaft and strain at one location on the beam, respectively. Comparing these results with the previous simulation in which the coefficient of restitution was updated as multiple impacts, with their lower velocities, occurred, one

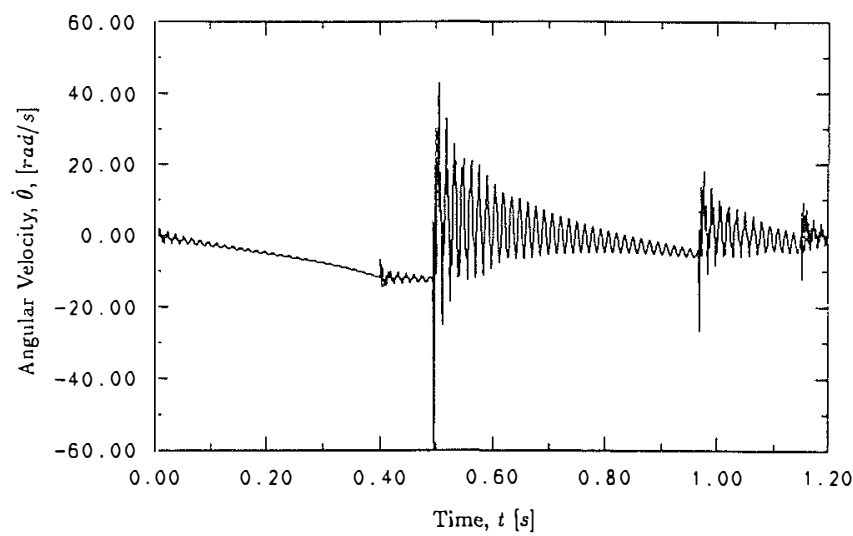

Fig. 9 Angular velocity of the rigid shaft, constant e

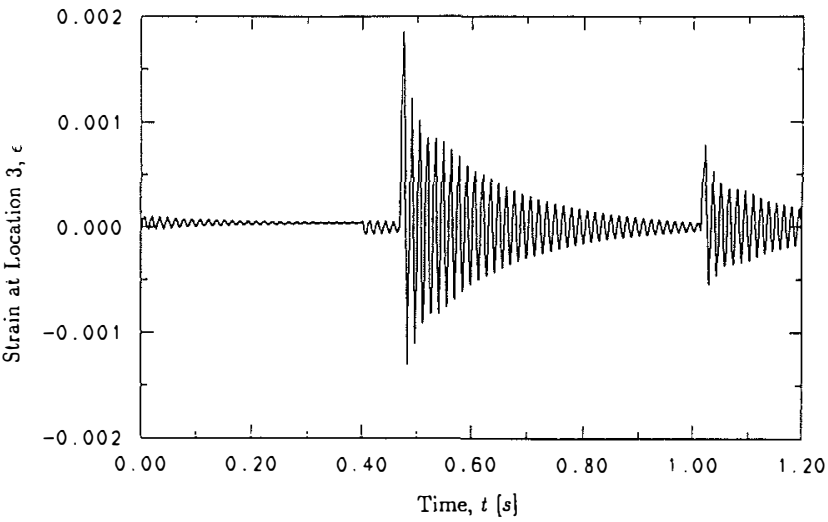

Fig. 10 Strain history at location 3, constant e

can conclude that the difference in the dynamic behavior is very minor. It can be concluded that as long as the value of the coefficient of restitution is appropriate for the first impact (which is responsible for the bulk of the energy loss), one can use the same value for the subsequent impacts.

A set of simulations and experiments were also performed for a steel and brass impact surface. In the simulations the value of $e$ for an aluminum-steel and aluminum-brass material pair was obtained using equation (30) of Yigit et al. (1988a). Torque inputs, which were obtained from the corresponding experiment for the steel and brass surface were used. Again, excellent agreement was found between the simulation and experimental results (see Yigit, 1988).

From these comparisons one can conclude that the momentum balance (coefficient of restitution) method can be used to predict the post impact dynamic behavior of similar systems with good accuracy. It must be noted, however, that the experiments performed in this study represent a particular configuration. In order to generalize the results some sensitivity studies were performed. These are presented in the following section.

\section{Sensitivity Studies of the Momentum Balance Method}

In Part 1 (Yigit et al., 1988a) an analytical investigation was made on a simple one degree of freedom system, and it was found that for most engineering systems the effect of flexibility on the coefficient of restitution would be negligible. The good agreement between theory and experiment reported above also supports this conclusion. To give these findings greater generality, some sensitivity studies will now be presented.

There are many possible measures of sensitivity. Here three different sensitivity measures are introduced as follows: 


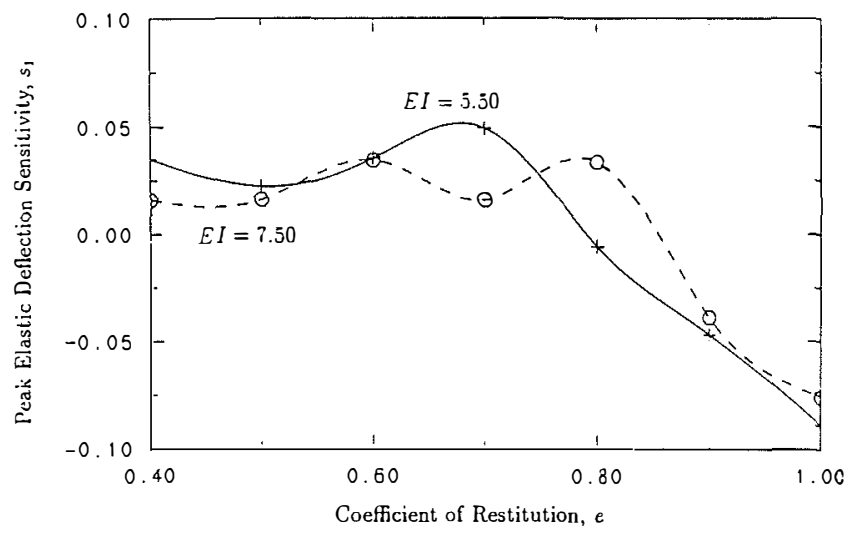

Fig. 11 Elastic peak deflection sensitivity, $s_{1}$

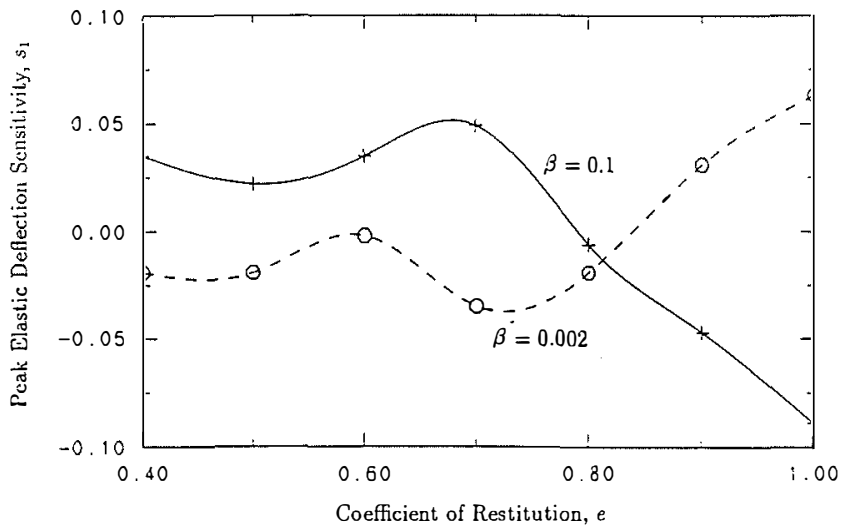

Fig. 12 Elastic peak deflection sensitivity, $s_{1}$

I Peak elastic deflection sensitivity, $s_{1}$ : This is basically a normalized peak deflection, and can be written as:

$$
s_{1}=\frac{w(l, t)_{\max }-\bar{w}(l, t)_{\max }}{\bar{w}(l, t)_{\max }}
$$

where $w(l, t)_{\max }$ are peak elastic deflections calculated for different values of $e$. $\bar{w}(l, t)_{\max }$ is the arithmetic mean of the $w(l, t)_{\max }$ values.

2 Tip displacement sensitivity, $s_{2}$ : Another possible measure for sensitivity is the total displacement $w_{p}$ due to both elastic and rigid body motion. Because of the rigid body motion, $w_{p}$ does not have a maximum value, and so replacing $w$ by $w_{p}$ in equation (4) is not appropriate. A sensitivity measure $s_{2}$ (which is a function of time is introduced by

$$
s_{2}=\frac{w_{p}(l, t)_{e=0.6}-w_{p}(l, t)}{w_{p}\left(l, t_{1}\right)_{e=0.6}}
$$

where $w_{p}(l, t)$ is the displacement corresponding to an arbitrary value of $e$, and $w_{p}(l, t)_{e=0.6}$ is the tip displacement for $e=0.6$. Here the value 0.6 is selected arbitrarily as an intermediate value of $e$ in the range of practical values. $t_{1}$ denotes a time after impact. In the sensitivity plots presented the $t_{1}$ is taken as the final time in simulations.

3 Global tip displacement sensitivity, $s_{3}$ : As a further measure the time integral of the absolute value of the sensitivity $s_{2}$ was used. This sensitivity measure is defined as:

$$
s_{3} \frac{\int_{t_{0}}^{t_{1}}\left|s_{2}\right| d t}{t_{1}-t_{0}}
$$

where $t_{0}$ is the time of the impact, and $t_{1}$ is a given time after impact.

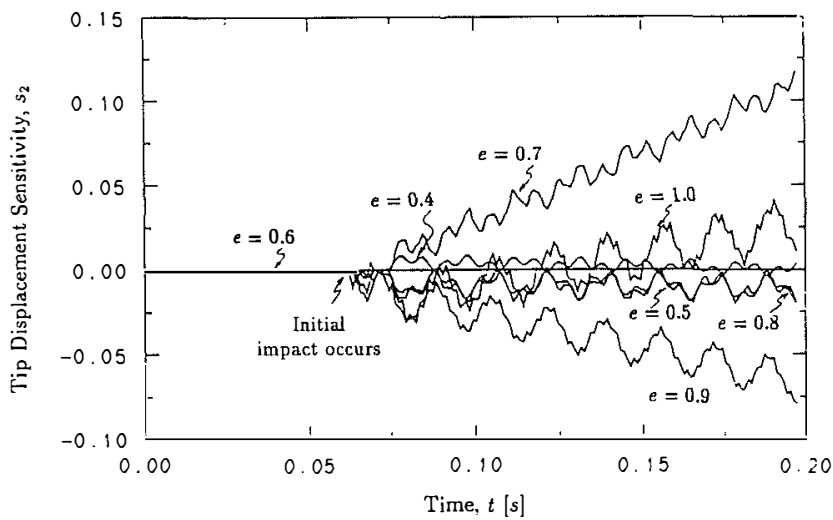

Fig. 13 Tip displacement sensitivity, $s_{2}$, for $E I=7.50$

Table 2 Global tip displacement sensitivity $s_{3}$, for $E I=7.5$

\begin{tabular}{|c|c|c|c|}
\hline \multirow{2}{*}{ Coefficient of } & \multicolumn{3}{|c|}{ Values of $s_{3}$ for } \\
\cline { 2 - 4 } Restitution, $e$ & $t_{1}=0.10$ & $t_{1}=0.15$ & $t_{1}=0.2$ \\
\hline 0.4 & 0.0034 & 0.0033 & 0.0028 \\
\hline 0.5 & 0.0059 & 0.0081 & 0.0089 \\
\hline 0.6 & 0.0 & 0.0 & 0.0 \\
\hline 0.7 & 0.0132 & 0.0335 & 0.0527 \\
\hline 0.8 & 0.0076 & 0.0089 & 0.0092 \\
\hline 0.9 & 0.0173 & 0.0280 & 0.0378 \\
\hline 1.0 & 0.0130 & 0.0104 & 0.0134 \\
\hline
\end{tabular}

Values of $s_{1}$ as functions of $e$ are given in Fig. 11 for two different flexural rigidity values. In both cases elastic peak deflections vary only by about 5 percent for a wide range of coefficient of restitution values in the practical range of 0.4 to 0.8 . Figure 12 shows $s_{1}$ for two different inertia ratios $\beta=\left(I_{R} / I_{B}\right)$. Again in both cases elastic peak deflections change only of the order of 5 percent for $e$ in the range of 0.4 to 0.8 .

Results using the $s_{2}$ sensitivity measure are presented in Fig. 13 for different values of $e$ and one value of flexural rigidity. In all cases the maximum value is about 10 percent up to the final time in the simulation. Similar small values of $s_{2}$ were found for other rigidity values.

The $s_{3}$ sensitivity results are presented in Table 2 for a particular flexural rigidity $(E I=7.50)$, and different $t_{1}$ and $e$ values. The maximum value of $s_{3}$ is less than 0.06 .

These results showing the relative insensitivity to variations in $e$ implies that, even if the flexibility and the system configuration does affect $e$, that may not significantly affect the response. Thus, it can be concluded that a coefficient of restitution value which is appropriately chosen from the rigid body data would give sufficiently accurate results, within engineering tolerances, for many situations of practical interest. By appropriately chosen we mean that it should be selected based on the expected initial impact velocity, and the material pair in question.

\section{Concluding Remarks}

In Part 2, we have described experimental and numerical 
studies on the dynamics of a flexible radially rotating beam with impact.

The momentum balance method has been demonstrated to be capable of accurately predicting the dynamics of systems which consist of both rigid and elastic links undergoing impact. This has been justified by a set of experiments performed on a specific system.

It was also shown that using a constant coefficient of restitution (provided that this value is appropriate for the first of the multiple impacts) can give almost identical results with those using a velocity dependent coefficient of restitution.

Sensitivity studies show that the model will also work reasonably well over a wide range of parameters covering most engineering situations. It can be concluded that if one is interested in the post impact dynamic behavior of the system, the momentum balance model and the coefficient of restitution concept can be used with reasonable confidence.

\section{Acknowledgments}

The authors would like to thank Mr. Gerald E. Lilly of
Eastman-Kodak Company for his help with the high speed video recordings, and $\mathrm{Mr}$. Steve Culp of The University of Michigan, for his assistance with setting up the experiments.

\section{References}

1 Goldsmith, W., 1960, Impact, Edward Arnold Publishers Ltd. 2 Hetényi, M., 1950, Handbook of Experimental Stress Analysis, John Wiley and Sons Inc.

3 Johnson, K. L., 1985, Contact Mechanics, Cambridge University Press. 4 Mason, H. L., 1935, "Impact on Beams," Trans. of the ASME, Vol. 58, pp. A55-61. $\mathrm{OH}$.

6 Yigit, A., Ulsoy, A. G., and Scott, R. A., 1988a, "Dynamics of a Radially Rotating Beam with Impact, Part 1: Theoretical and Computational Model," ASME Journal of Vibration and Acoustics, Vol. 112, No. 1, pp. 65-70.

7 Yigit, A., Ulsoy, A. G., and Scott, R. A., 1988b, "Flexural Motion of a Radially Rotating Beam Attached to a Rigid Shaft," Journal of Sound and Vibration, Vol. 121, pp. 201-210.

8 Yigit, A., 1988, Dynamics of a Radially Rotating Beam with Impact: Implications for Robotics, Ph. D. Dissertation, The University of Michigan. 